\title{
The Space Information Content of Wechat with Its Application for High Education Based on Big Data
}

\author{
Chunfen $\mathrm{Bu}^{1, \mathrm{a}^{*}}$ \\ ${ }^{1}$ Department of physical science and technology, Kunming University \\ a18459423@qq.com \\ *The Corresponding Author
}

Keywords: Big data; WeChat; Public Security system; Space content

\begin{abstract}
The key purpose of this paper aims to discuss the possibility of constructing the public information system based on the Big data for the instruction based on WeChat. The big data is introduced firstly and its development is also discussed. Then the public security pattern of WeChat is proposed and is referred to as the space content. The relationship between public security and the WeChat is discussed subsequently. At last, in order to illustrate our theory, some experiments are constructed to testify the corresponding theory. The analysis and discussion indicate that the construction of public security system based on Big data is significant to the instruction with WeChat and the corresponding technology is mature.
\end{abstract}

\section{Introduction}

In Mid-19th century, the steam engine opened the time called " the first industrial revolution", which take the human world into the path of rapid development. On the early 20th century, the utilization of electricity promoted the Second Industrial Revolution. At the same time, the natural sciences, especially the development of mathematics and statistics, the fact that the human society can be described by a mathematical model come true. Meanwhile, the development of technology has also brought social and cultural developing rapidly, with forming a large-scale "knowledge explosion. Based on this fact, the rapid development of modern university education is improving the level of human culture fundamentally. At the latest 20th century, the development of modern computers and electronics have brought new historical leap in human society. In this time, the word "Digital" became a human familiar vocabulary. Then, Internet has become the main platform for data exchange, which makes the distance between countries being shortened. Furthermore, culture, information, intelligence and even passing messages has no longer been subject to geographical factors, which greatly reduce the human speed accessing to information. Therefore, the word "Data" has become a strategic resource for governments attaching great importance [1]. For example, the "information superhighway" proposed by USA government aims to improve the development of the national strategic level.

However, when the wheel of history are role into the 21 st century, human society is facing a new opportunity, perhaps, this is a new opportunity to say --- "big data". Narrowly speaking, the term big data refers to the mass storage for data. Oppositely, the Big Data implies the information harvesting by using mathematics, theoretical statistics and the high-speed computer from large databases [2]. By analyzing data patterns hiding in data, he possibility one thing will happen can be predicted. Actually, on the first 10 years of the 21 st century, human society is the era of data explosion with the popularity of mobile computing platforms, the birth of high-performance computing equipment, the timing of the development of modern statistics, information management platform for big data research in an era ripe. Currently, there are many companies use "big data," it is the opportunity to expand their business by obtaining complete profit model transformation and to bring amazing profit growth [3]. Such as the famous Amazon, Facebook and so on.

On the other hand, the big data also play more role for the police information construction $[4,5]$. For example, the "three-building" project, it relies on the development of the technology. One of the face of the impact of big data era is the technology. Meanwhile, both the challenge and the 
opportunity exist. The current status of public security information technology, briefly speaking, can only achieve "digital" level, which is far from the "data" of the hierarchy. Despite the utilization of the universal application of the office automation system, the "police mechanized" platform, large information systems, information management systems can only help the easy analysis for the public security information. Furthermore the current management information system (MIS or Expert Decision System) can only facilitate the daily police work, for data management is only completed junior record store. The more complex systems, such as large information systems, data can be obtained by only some simple pattern recognition, or by making some simple decisions. The potential value of the data for the current public security information system is not fully utilized. In the era of big data, data mining methods is used to excavate the information and the results are translated to the expert system[6]. In other words, decision theory system used in the digital age is not suitable for large data mining information. To build large data public security information system, we need to study the new data modeling, analysis, forecasting strategy mode. The study of these contents relies on having a Master Model for guidance. Therefore, Public Security Information construction under the Big Data era is important for future work. In this paper, the possibility for constructing the big data application for police security is discussed.

\section{The Significance of the Construction of Big Data for Public Security Application}

Big data processing mode is important for speeding up the construction of public security information. The context of data using a large data analysis and decision-making ability and the public security organs can be more convenient to access to the intelligence sources, it can also be completed quickly merge need to use the clues. At the same time, large data system can also help a clearer understanding of the public security organs trends humanistic nature of specific regions. Therefore, in terms of many aspects of public security work, public security information technology can build large data directly which is related to the future of public security work carried out efficiently. Furthermore, the public security organ of intelligence information can also improve the access speed of trailing information. In details, the significance lies in the following aspects:

Firstly, the basic functions of large data systems can provide quick access to information from massive data distributed. In history, especially in 19th century, the Rothschilds took advantage to build their own rapid intelligence network in advance to detect the news of the defeat of Napoleon, which was able to earn in the London financial markets pours [1]. In the 20th century, the Amazon use their textbook to grasp of user record information and to recommend books to readers completed the creative work and ultimately success. Of course, the big data not only can provide business services. For public security work, big data applications can also play a role to analysis, decision-making by using a large data. Investigating techniques will greatly expand the public security organs.

Secondly, relying on large data analysis and decision-making, the public security organs to quickly analyze clues, locked the suspect. From the information theory point of view, investigation, interrogation, investigation and other works conducted by the public security organs in theory is to make information (uncertainty) criminal event contains a minimum. It has been put into the public security information system used to some extent and to accelerate the realization of the above process. However, due to the small size of the dataset, we can rely on clues intersection method to look for clues. This approach by seeking event, then the intersection of character attributes and the accurate information will determine clues. However, finding the cross point is not an easy thing in the complex set of clues. For large data, due to the large amount of data, it often does not require precise intersection analysis as a basis for relations. 


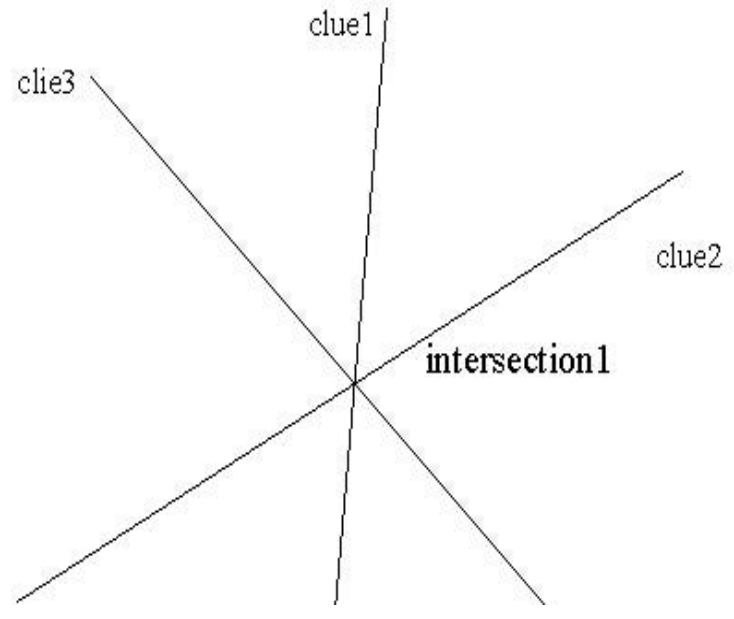

(a) Traditional analysis based on clues

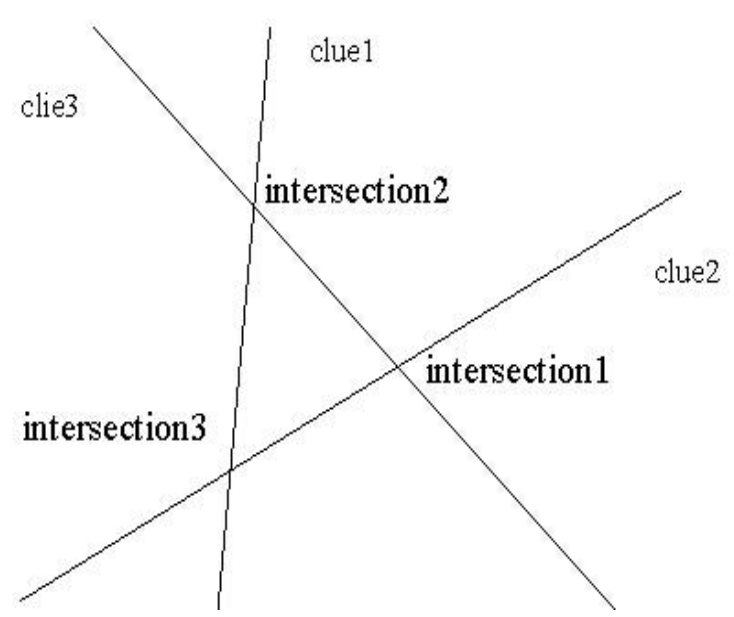

(b) Analysis clues based on Big data

Figure 1. The sign of two analysis methods based on traditional way and big data respectively

From Fig. 1, the more clues space are constituted. In order to obtain useful information cross, it must complete multi-threaded with multi-line indication line level crossings. On the other hand, however, data from multiple clues of the cube space plane may be in the form of the intersection to form a support plane estimate. Thus such a way that the estimated gain clues which is better than the era when small data existed can be more easier. At the same time, with an estimated decision-making capacity, it often can get more than a small data sets to obtain more useful clues message. It also implies that the large public security information system should take more advantage of the current information system.

Finally, the big data can be more truly reflect the relationship between people and the analyze trends of Social Relations particular environment. Currently, the public security information system based on the relational model uses a two-dimensional table structure (relational database using a two-dimensional table describes the data association) to describe the community of people attribute and relationship with each other. The results described in this way brings a loss of information associated with the space, and it relies on a large-scale relational database for querying processing statements in order to obtain the required information. In contrast, large data management results are described by using a spatial grid which enable the multiple spatial pattern (multiple threads get way) to describe the information obtained. For example, for constructing the analysis model to obtain the citizens' basic information, such as letters and other messages, the interpersonal relationships extending from the relationship diagrams can make predictions for the region's population with more activity. Meanwhile, it is important to avoid mass incidents.

\section{The Space content}

Actually, for most mobile application relying on the public security system, such as WeChat, the information pattern is similarly illustrated with Fig 1 . Let ${ }^{N}$ denote the number of items which are joining in calculating the corresponding information content. Each item is consist of any counting events which is drew by using some special statistic approaches. Obviously, one item can be described by a directed vector. $\mathbf{V}_{\mathbf{i}}$ denoted here. Let the direction vector ${ }^{\mathbf{D}_{\mathbf{i}}}$ denote the directions of the elements contained in $\mathbf{v}_{\mathbf{i}}$. It is easy to find that, the directed vector $\mathbf{v}_{\mathbf{i}}$ is given in (1)

$$
\mathbf{V}_{\mathbf{i}}=\left\{C_{1}, C_{2}, \ldots, C_{N}\right\}
$$

Each $^{C_{i}}$ denote one counting vector which come from the exact application. For example, for instruction, ${ }^{C_{i}}$ can be considered as the quantity of the instruction drew with: Good, Normal and Bad. 
Then the direction vector ${ }^{\mathbf{D}_{\mathbf{i}}}$ can be represent as (2):

$$
\mathbf{D}_{\mathbf{i}}=\left\{\theta_{1}, \theta_{2}, \ldots, \theta_{N}\right\}
$$

Where each ${ }^{\theta_{i}}$ denote the direction of the corresponding counting vector.

Based on this discussion, to calculate the information of the public system directly is difficult. There are more than 3-D vectors. Inspiring by Fish Information theory, the corresponding information content can be calculated with reducing domaintion. So we first give the calculation of information content for each ${ }^{C_{i}}$ as (3)

$$
L_{1}=S_{1} \log S_{1}-n_{0} \log n_{0}-n_{1} \log n_{1}-n_{2} \log n_{2}-\frac{1}{2} \log \frac{S_{1}}{n_{0} n_{1} n_{2}}+\sigma
$$

Where ${ }^{S_{1}}$ denote the total number of counts contained in ${ }^{C_{i}}$ and ${ }^{m_{i}}$ denote the number of counts for each case.

Actually, the information content can be represented by using the corresponding description length $[7,8]$, it is also $L_{i}$ in (3).

Then the Fish information can be calculated by (4) when each ${ }^{C_{i}}$ is reformed as (3)

$$
\zeta=\mathbf{V}_{\mathbf{i}} \bullet \mathbf{D}_{\mathbf{i}}
$$

Then parameter $\zeta$ is the space content in this paper. Its utilization is to testify the estimation accuracy of a public system under big data.

For instruction based on WeChat, each issue for one topic can be considered as a ${ }^{C_{i}}$, its direction can be represented as ${ }^{\cos \theta_{i}}$. Then its space content can be calculated by (4).

If the space content is small, its estimation accuracy is high.

In next section, the WeChat platform is constructed for instruction. Then its corresponding space content is calculated.

\section{Experiments}

In order to illustrate the proposed theory, some experiments are constructed.

Firstly, the WeChat platform is constructed.

The corresponding WeChat platform is given in Fig. 2:

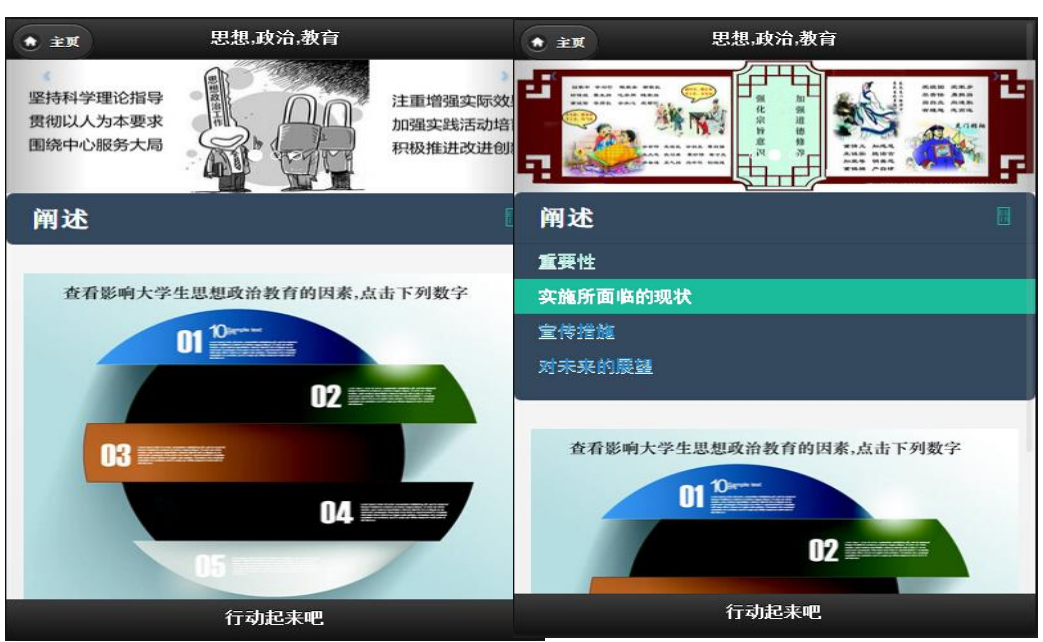

Figure 2. The application of our instruction system based on Wechat

In this platform, there are two issues to show some messages for instruction. There are two voting systems which are corresponding to these two issues respectively. Actually, the purpose of this experiment is to testify the proposed theory, so that, the results are given here firstly. 
There are two cases for this experiment. We discuss the two issues first.

Issue 1: The math equation resolving. Right: $80 \%$ Wrong: $20 \%$

Issue 2: The history questions. Right: $75 \%$ Wrong $25 \%$.

Two cases:

Case 1: The radio that the students answer these two group questions and the results is similar.

Case 2: The radio that the students answer these two group question and the result is not similar.

Actually, the different radio is reasoning in the security of public system under more error code or noise.

Then we will calculate the corresponding space content respectively. The results are listed in Table 1:

Table 1 The comparison of two case with space content

\begin{tabular}{|c|c|c|c|}
\hline case & Issue 1 & Issue 2 & Space content (bits) \\
\hline 1 & 22882.4 & 23322.8 & 21222.5 \\
\hline 2 & 23392.3 & 31303.1 & 33435.2 \\
\hline
\end{tabular}

It is obviously that if two cases are similar, the corresponding space content is smaller than the case that two cases are not similar. Meanwhile, the exact results is similar with the calculated results, which implies that the proposed theory and method can be used to illustrate the public security system and it can also be used to testify the instruction under big data.

\section{Conclusion}

The analysis and discussion indicate that the construction of public security system based on Big data is significant to the instruction with WeChat and the corresponding technology is mature.

\section{Acknowledge}

This work is supported by Science Foundation of Kunming University under Grant (XJL14003) and this work is inspiring by PH.D Chen.

\section{References}

[1] Viktor. M. Schönberger, Kenneth Cukier, A Revolution That Will Transform How We Live, Work, and Think, Houghton Mifflin Harcourt, 2013.

[2] Asur, Sitaram and Bernardo A.Huberman, "Predicting the Future with Social Media.", Proceedings of the 2010 IEEE/WIC/ACM International Conference on Web Intelligence and Intelligent Agent Technology, 2010, pp.492-499.

[3] Lee, YH., Hsieh, Y, Adding Innovation Diffusion Theory to the Technology Acceptance Model: porting Employees' Intentions to use E-Learning Systems[J]. Educational Technology\& Society, 14 (4), 2011:124 -137

[4] Hsin-Chang Yang, hung-Hong Lee. 2005. A text mining approach for automatic construction of hypertexts. Expert Systems with Applications, 9:723-734.

[5] Schweitzer F, Fagiolo G, Sornette D, Vega-Redondo F, Vespignani A, et al. (2009) Economic Networks: The New Challenges. Science 325: 422-425.

[6] Bapler.P\&Murdoch, Academic Analytics on Data Mining in Higher Education. Intemational Jounlal for the Scholarship of Teaching and Leanling, 4(2), 2013.

[7] Min Chen etc, Context Quantization Under the Minimum Increment of the Adaptive Code Length[C], proceeding of the information Technology IEEE, 2013, HongKong : 9-12.

[8] Min Chen, Jianhua Chen, Affinity Propagation for the Context Quantization[J], Advanced Materials Research, vol(791), 2013: 1533-1536. 